\title{
Merlin and ezrin get organized
}

The ezrin, radixin and moesin (ERM) proteins and the closely related tumour suppressor neurofibromatosis type 2 (NF2; also known as merlin) function as scaffolds to organize the cell cortex and to help regulate cell polarity during epithelial morphogenesis, a process that is often disrupted in tumorigenesis. However, how NF2 and the ERM proteins achieve this organization, and the functional relationship among these proteins, is not completely clear.

Andrea McClatchey and colleagues followed the localization of ezrin and NF2 during the establishment of apical-basal polarity in single Caco2 intestinal epithelial cells embedded in Matrigel. They noted that ezrin became restricted into a cap-like structure at the plasma membrane prior to the first cell division. The cells were uniformly surrounded by extracellular matrix and the cap lacked markers of apical polarity, indicating that intrinsic cues establish this ezrin localization prior to the formation of a true apical surface. Furthermore, the ezrin cap began to form during G1 phase of the cell cycle and was mostly completed by $S$ phase, and cap formation required the cyclin-dependent kinase CDK5 (which can phosphorylate the ezrin FERM domain), linking this event to the cell cycle.

In contrast to ezrin, NF2 did not localize to the cap structure, but was found throughout the cell cortex. However, short hairpin RNA (shRNA) against NF2 prevented ezrin cap formation, and resulted in ectopic

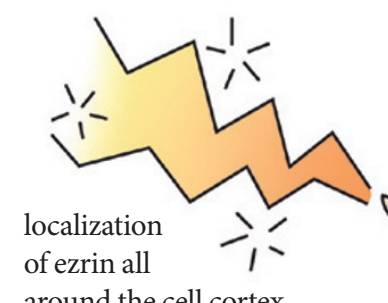

around the cell cortex.

Therefore, NF2 seems to

be involved in restricting the localization of ezrin, a process that the authors found requires a-catenin; this is interesting as it indicates a junction-independent function for $\alpha$-catenin.

Given the connections of the ezrin cap to the cell cycle, the authors also examined the centrosomes and mitotic spindle position in single Caco 2 cells; they found that their localization was tightly correlated with the ezrin cap. Supporting this, centrosomes in cells expressing NF2 shRNA were found near areas of the cortex with ectopic ezrin, and spindles were aberrantly oriented in these cells. Through organized cell division, Caco 2 cells normally form polarized cysts with single lumen, a process that requires correct spindle orientation. Cells lacking NF2 or a-catenin formed aberrant structures with multiple lumens, a phenotype that was previously observed in other models with misorientation of the mitotic spindle.

To determine the in vivo relevance of these findings, the authors examined three tissues in $\mathrm{Nf2} 2^{-/-}$mice. They found that $N f 2^{-/-}$mice have spindle orientation defects and multi-layering in the skin, aberrant centrosome localization in the kidney

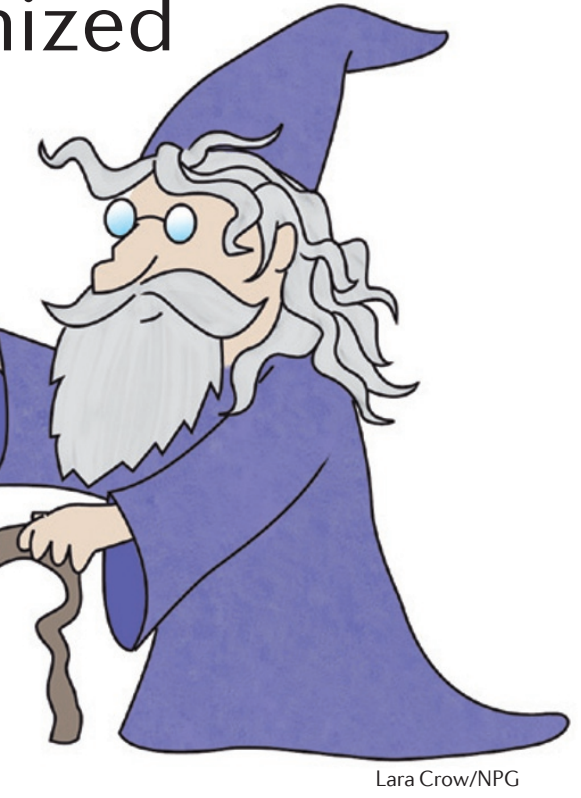

(these mice eventually develop renal carcinomas), and ectopic ezrin localization and multi-layering in the colon. In addition, cancer cells often have extra centrosomes; these can be clustered to allow the cell to form bipolar spindles, but loss of clustering can lead to multipolar spindles and aneuploidy. In several tumour cell lines expressing NF2 shRNA, as well as NF2-deficient mesothelioma cells, loss of NF2 prevented centrosome clustering and resulted in multipolar spindle formation. Therefore, NF2 may suppress tumours by restricting ezrin localization to establish and maintain cell polarity and spindle orientation, and may also help prevent genomic instability.

Sarah Seton-Rogers

ORIGINAL RESEARCH PAPER Hebert, A. M. et al. Merlin/ERM proteins establish cortical asymmetry and centrosome position. Genes Dev. 26, 2709-2723 (2012) 